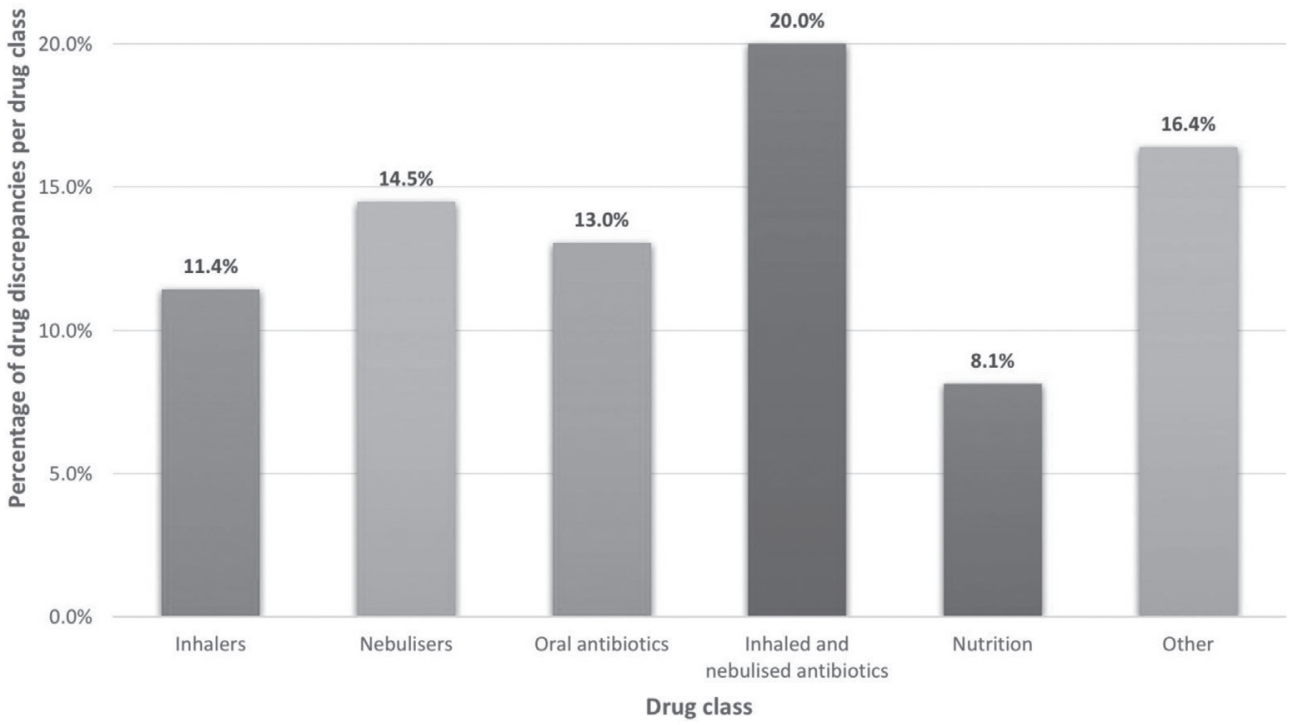

Abstract P99 Figure 1 Drug discrepancy breakdown by class

\section{P100 PRE-TRANSPLANT C-REACTIVE PROTEIN (CRP) AS A MARKER OF POST-TRANSPLANT OUTCOMES IN PATIENTS WITH CYSTIC FIBROSIS (CF)}

A Fazleen, J Parmar. Papworth Hospital NHS Foundation Trust, Cambridge, UK

\subsection{6/thoraxjnl-2016-209333.243}

Introduction Although novel therapies for CF have been introduced, the condition still has a high rate of complications and early death. Transplantation remains the only hope for extended survival and quality of life. Our study aimed to determine whether pre-transplantation CRP could predict outcomes posttransplantation.

Methods In a retrospective analysis of all lung transplantations performed at our centre between 2001 and 2016, only patients who had complete data were analysed. CRP levels within 72 hours pre-transplantation were compared with length of intubation, ITU and hospital stay, Primary Graft Dysfunction (PGD), pulmonary infection within 3 months post-transplantation and rates of acute cellular rejection.

Results Among 100 patients who underwent lung or heart/lung transplantation, 72 patients had a complete dataset. Average age at transplantation was 28.1 in both groups.

48 patients had CRP $<40 \mathrm{mg} / \mathrm{L}$ (24 males, 24 females, median CRP 14), and 24 patients had CRP $>40 \mathrm{mg} / \mathrm{L}$ (14 males, 10 females, median CRP 60). Average age of donor was 41.6 in the low CRP group, and 41.8 in the high CRP group.

Average duration of intubation was 66.7 hours in the low CRP group, versus 48.0 hours in the high CRP group, while average length of ITU stay was 6.9 days in the low CRP group, versus 9.3 days in the high CRP group (p 0.76). Average duration of hospital stay was 26.3 days in the low CRP group, and 25.7 days in the high CRP group. Mean number of infections in the low CRP group was 2.4, and 3.3 in the high CRP group (p 0.52). Mean number of episodes of rejection was 0.4 in the low CRP group, and 0.6 in the high CRP group (p 0.36).

Kaplan-Meier plots showed no evidence of impact on survival to 5 years.

Conclusion CRP within 72 hours pre-transplantation in patients with CF was not a predictor of short- or long-term outcomes in our study. It is important to note that the sample size for this study was quite small, possibly contributing to this finding. Extension of this study to include patients from multiple transplant centres would be beneficial.

\begin{tabular}{llll} 
Abstract P100 Table 1 & \multicolumn{2}{l}{} \\
\hline Postoperative data & Average & Average & \\
\hline & $<40(\mathrm{n}=48)$ & $\geq 40(\mathrm{n}=24)$ & p.value \\
PGD & 4 & 0 & 0.29 \\
Intubation time (hours) & 66.7 & 48.0 & 0.52 \\
Blood loss in first 24 hours (mls) & 1255.0 & 1550.8 & 0.19 \\
Length of ITU stay (days) & 6.9 & 9.3 & 0.76 \\
Length of hospital stay (days) & 26.3 & 25.7 & 0.50 \\
Renal failure & 2 & 1 & 1.00 \\
Airway complications & 4 & 1 & 0.77 \\
Rejection episodes & 0.4 & 0.6 & 0.36 \\
Infection episodes & 2.4 & 3.3 & 0.52 \\
1 year survival & & & 0.18 \\
\hline
\end{tabular}

\section{Improving Lung Cancer Care and Outcomes}

\section{P101 STRUCTURED HOLISTIC NURSING ASSESSMENT (HNA) IN BUCKINGHAMSHIRE HEALTHCARE TRUST (BHT) FOR LUNG CANCER \& MESOTHELIOMA PATIENTS: PROCESS AND FINDINGS}

J Mowforth, H Steiner, A Prasad. Buckinghamshire Healthcare NHS Trust, Aylesbury, UK

\subsection{6/thoraxjnl-2016-209333.244}

Aim Lung cancer and mesothelioma patients have complex needs. Without a structured process of screening and assessment these can go undetected. Structured screening and assessment needs considered planning. Our experience of screening and assessment of the holistic needs for lung cancer and mesothelioma patients in BHT is described.

Method All BHT lung cancer and mesothelioma patients are invited to be screened post diagnosis using the Distress Thermometer and Needs checklist (Brennan et al 2012). ${ }^{1}$ Participation 
rates vary between 55-65\% and since 2013, 467 patients have been contacted with 265 patients participating. A database stores all HNA activity. This includes individual overall numerical score for distress (0-10), a breakdown of categories of need including physical, emotional, practical, social and spiritual. It also records specific aspects of distress within each category and where specified the top four aspects of need.

Results Of the 265 respondents, 196 (74\%) recorded physical problems, 145 (54\%) recorded emotional issues with worry, fear, sadness and depression sited as the top four aspects. In addition $63(24 \%)$ reported practical problems e.g. money and transport, $53(20 \%)$ family problems, and $32(12 \%)$ spiritual concerns. Overall tiredness, breathing, getting around and worry are the top four needs recorded by our patients. Of all patients screened to date a third score Distress above 5, these patients are prioritised for a nurse led clinic.

Conclusion Through screening issues often not disclosed in doctor led clinics are identified and addressed leading to involvement of other professionals and services. Screening patients away from the clinical setting enables greater disclosure, prevents bias or influence on scoring and allows complex needs that might go undetected to be explored. The process enables care to be patient focused and proactive in managing complex needs before crisis events occur.

\section{REFERENCE}

1 Brennan J, et al. Refinement of the distress management problem list as the basis for a holistic therapeutic conversation among UK patients with cancer. PsychoOncology 2012;21:1346-56.

\section{P102 \\ THE LIVERPOOL HEALTHY LUNG PROJECT - SEEKING OUT EARLY STAGE LUNG CANCER}

${ }^{1} \mathrm{MJ}$ Ledson, ${ }^{2} \mathrm{~S}$ Grundy, ${ }^{3} \mathrm{E}$ Gaynor, ${ }^{3} \mathrm{R}$ Arvanitis, ${ }^{3} \mathrm{M}$ Timoney, ${ }^{4}$ J Field. ${ }^{1}$ Liverpool Heart and Chest Hospital, Liverpool, UK; ${ }^{2}$ Aintree Hospital, Liverpool, UK; ${ }^{3}$ Liverpool Clinical Commissioning Group, Liverpool, UK; ${ }^{4}$ Liverpool University, Liverpool, UK

\subsection{6/thoraxjnl-2016-209333.245}

Liverpool has one of the highest respiratory morbidity rates in England, with double the lung cancer incidence, particularly in lower socioeconomic groups. To tackle this health inequality in February 2016 in conjunction with Liverpool CCG, the primary care sector, public health and Liverpool University we commenced the 3-year $£ 3.3 \mathrm{M}$ Liverpool Healthy Lung Project. The project has 2 sequential phases.

Phase 1 By means of a series of coordinated focused public engagement events throughout the city, starting in areas with higher lung cancer incidence, this aims to promote positive messages around lung health, and address the fear and fatalism surrounding lung cancer.

Phase 2 All individuals between the ages of 58-70 who have COPD, have smoked, or are asbestos exposed are invited to a face to face lung health check conducted by an experienced respiratory nurse. Positive lifestyle messages are promoted and a 5year personal lung cancer risk calculated (www.MyLungRisk.org using LLPv2 risk model). Those who trigger the 5\% threshold are offered a low dose thoracic CT scan.

The community healthy lung events attracted 1346 interactions and 462 individuals completed spirometry. 90 (19\%) of these tests were abnormal which triggered a consultation in primary care.

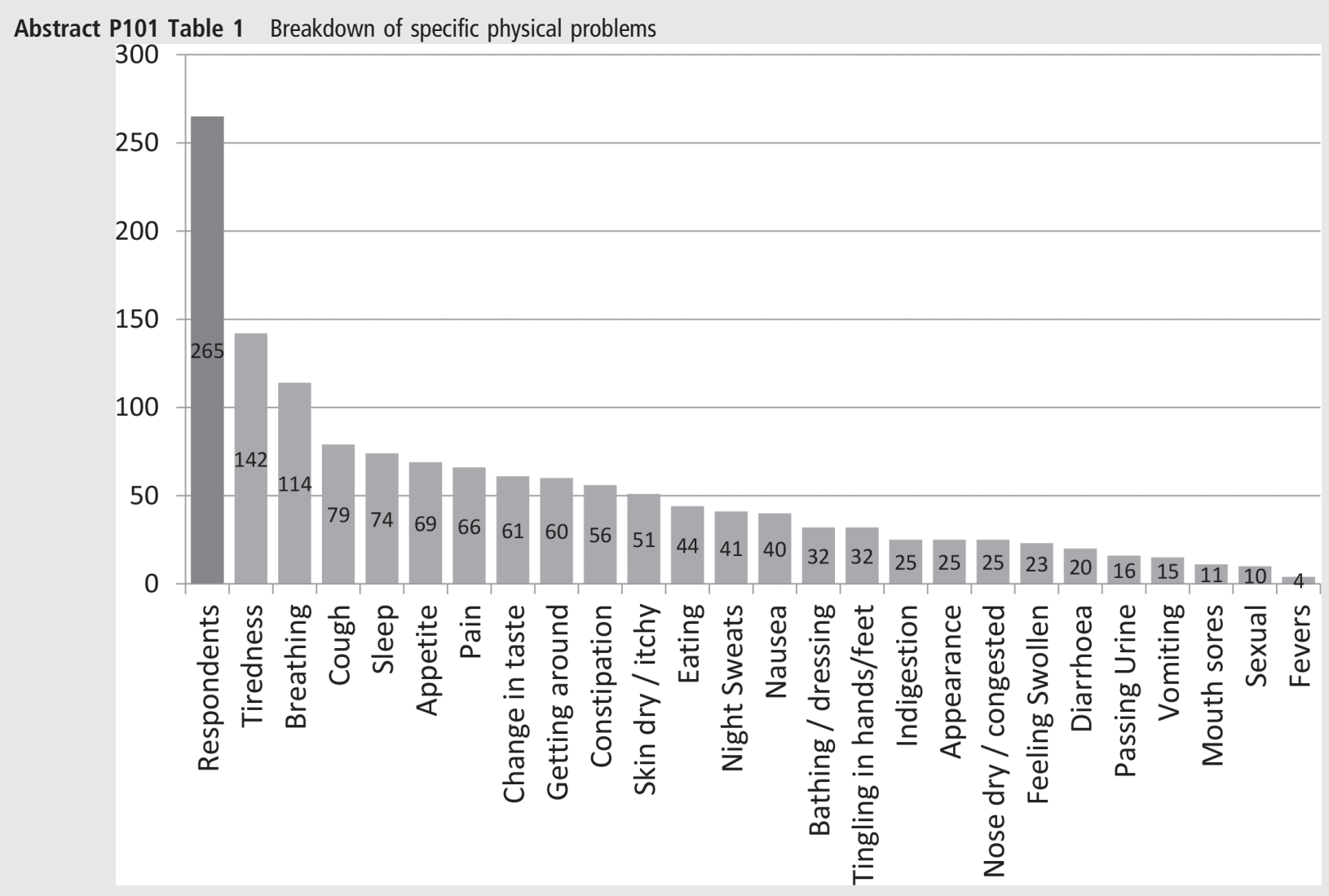

